\title{
Whole genome annotation and comparative genomic analyses of bio-control fungus Purpureocillium lilacinum
}

\author{
Pushplata Prasad ${ }^{*}$ D, Deepti Varshney and Alok Adholeya
}

\begin{abstract}
Background: The fungus Purpureocillium lilacinum is widely known as a biological control agent against plant parasitic nematodes. This research article consists of genomic annotation of the first draft of whole genome sequence of $P$. lilacinum. The study aims to decipher the putative genetic components of the fungus involved in nematode pathogenesis by performing comparative genomic analysis with nine closely related fungal species in Hypocreales.
\end{abstract}

Results: de novo genomic assembly was done and a total of 301 scaffolds were constructed for $P$. lilacinum genomic DNA. By employing structural genome prediction models, 13, 266 genes coding for proteins were predicted in the genome. Approximately $73 \%$ of the predicted genes were functionally annotated using Blastp, InterProScan and Gene Ontology. A $14.7 \%$ fraction of the predicted genes shared significant homology with genes in the Pathogen Host Interactions (PHI) database. The phylogenomic analysis carried out using maximum likelihood RAxML algorithm provided insight into the evolutionary relationship of $P$. lilacinum. In congruence with other closely related species in the Hypocreales namely, Metarhizium spp., Pochonia chlamydosporia, Cordyceps militaris, Trichoderma reesei and Fusarium spp., P. lilacinum has large gene sets coding for G-protein coupled receptors (GPCRs), proteases, glycoside hydrolases and carbohydrate esterases that are required for degradation of nematode-egg shell components. Screening of the genome by Antibiotics \& Secondary Metabolite Analysis Shell (AntiSMASH) pipeline indicated that the genome potentially codes for a variety of secondary metabolites, possibly required for adaptation to heterogeneous lifestyles reported for $P$. lilacinum. Significant up-regulation of subtilisin-like serine protease genes in presence of nematode eggs in quantitative real-time analyses suggested potential role of serine proteases in nematode pathogenesis.

Conclusions: The data offer a better understanding of Purpureocillium lilacinum genome and will enhance our understanding on the molecular mechanism involved in nematophagy.

Keywords: P. lilacinum, de novo assembly, Whole genome sequencing, Annotation, Pathogen-host interaction, Phylogenomic analysis

\section{Background}

Purpureocillium lilacinum, previously named as Paecilomyces lilacinus, belongs to the fungal order Hypocreales that harbors species known to produce a diversity of secondary metabolites and bio-actives [1]. P. lilacinum produces paecilotoxin and is considered as a significant biological control agent against plant parasitic

\footnotetext{
* Correspondence: pushplata.singh@teri.res.in

TERI Deakin Nanobiotechnology Centre, TERI Gram, The Energy and Resources Institute, Gual Pahari,Faridabad Road, Gurgaon, Haryana 122 001, India
}

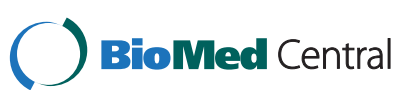

(c) 2015 Prasad et al. Open Access This article is distributed under the terms of the Creative Commons Attribution 4.0 International License (http://creativecommons.org/licenses/by/4.0/), which permits unrestricted use, distribution, and reproduction in any medium, provided you give appropriate credit to the original author(s) and the source, provide a link to the Creative Commons license, and indicate if changes were made. The Creative Commons Public Domain Dedication waiver (http://creativecommons.org/publicdomain/zero/1.0/) applies to the data made available in this article, unless otherwise stated. nematodes, especially economically important species of Meloidogyne incognita [2, 3]. Diseases caused by plantparasitic nematodes are considered as one of the major threats for global food security [4-7]. Nematode infection in economically important crops results in an estimated annual loss of US $\$ 100$ billion [8]. Research on nematodesuppressive soils has revealed a substantial presence of nematophagous fungi P. lilacinum and Pochonia chlamydosporia $[9,10]$. Similar to other species in Hypocreales, P. lilacinum has a broad host range and its various strains parasitize different species of nematodes and insects [11]. 
In addition, endophytically-colonized $P$. lilacinum has been recently reported to adversely affect reproduction of cotton-aphids putatively by inducing systemic response in plant [12].

The fungal order Hypocreales consists of plantpathogens, insect-pathogens, nematode-pathogens, plantendophytes and mycoparasites. These fungi are multitrophic in nature and several transitions between lifestyles have been predicted in their evolutionary history [13-15]. P. lilacinum is a ubiquitous soil hyphomycete and carry out saprophytic activities in varied habitats including agricultural fields, forests, grassland, deserts and estuarine sediments. Different isolates of $P$. lilacinum are found resistant to a wide range of temperature and $\mathrm{pH}$ [16]. On the other hand, P. lilacinum is also reported to exercise parasitic or endophytic lifestyles in the presence of a host organism such as nematodes, aphids and cotton plants (Gossypium hirsutum) [9-12].

P. lilacinum infects eggs and females of Meloidogyne spp. and causes death of the nematode embryos in 5 to 7 days [17]. A strain of P. lilacinum, strain 251, is now an active ingredient in several commercial bionematicides [18]. However, the molecular basis of the pathogenic mechanism employed by $P$. lilacinum against nematodes has been meagerly elucidated till date. Whole genome sequencing efforts of other Hypocreales fungi $[1,13,19-22]$ have revealed that these genomes code for an array of hydrolytic enzymes, secondary metabolites/ bioactives, non-ribosomal protein synthetases (NRPS) and polyketide synthetases (PKS) that are essential for their bio-control attribute. Experimental evidences show that extracellular hydrolytic enzymes including proteases, collagenases and chitinases are involved in the degradation of egg-shell components by P. lilacinum [23, 24]. Despite of strong prospect of secondary metabolites and hydrolytic enzymes produced by P. lilacinum in biological control of phyto-pathogens, only one gene encoding for serine protease [23] and one encoding for keratinase [16] have been molecularly characterized so far.

Considering the increasing demand for alternative plantpathogen management approaches in agricultural systems, we carried out whole genome annotation and comparative genomic analyses of the nematophagous fungus $P$. lilacinum. The study provides new insights into the genome of this commercially important biological control agent. The work would pave way for understanding important pathways and genes utilized by $P$. lilacinum to carry out nematophagous and endophytic activities, which is expected to aid in developing methods to protect crop plants.

\section{Results and discussion Global genome structure}

The $P$. lilacinum genome was sequenced to $200 \mathrm{x}$ coverage and by mapping the reads to contigs, the genome sequence was assembled into 301 scaffolds with total gap size of 526,913 bases. The sum of the scaffolds length is equal to $40.02 \mathrm{Mb}$. The longest scaffold length was of 3.7 Mb. The N50 and N90 values were 1,827,308 bp and 93,833 bp respectively. The predicted assembly size was $44.76 \mathrm{Mb}$ by $\mathrm{k}$-mer analysis with the best $\mathrm{kmer}$ value $=51$ (Additional file 1: Figure S1). The MAKER annotation pipeline [25] predicted 13,266 protein coding genes, which is comparatively more than other species belonging to entomopathogenic or mycoparasitic families of Hypocreales. Core Eukaryotic Genes Mapping (CEGMA) [26, 27] analysis against a set of 248 conserved protein families that occur in a wide range of core eukaryotic gene datasets (CEGs) http://korflab.ucdavis.edu/Datasets /genome completeness/index.html\#SCT2) found $96.77 \%$ of the core genes were matched, indicating that the draft genome sequence of $P$. lilacinum was largely complete (Additional file 1: Table S1). tRNAScan-SE $[28,29]$ predicted a total of 91 tRNAs in the genome. The average gene density (303/ $\mathrm{Mb})$ and the average gene length $(1.51 \mathrm{~Kb})$ of $P$. lilacinum are similar to other Ascomycetous fungi [13]. The genome size $(40.02 \mathrm{Mb})$, total number of predicted genes $(13,266)$ and the \%GC $(58.57 \%)$ content of P. lilacinum are comparable to the nematophagous fungus Pochonia chlamydosporia $(41.0 \mathrm{Mb})$ of Hypocreales (Table 1 and Additional file 1: Table S2). When compared with other fungal orders, the genome size of $P$. lilacinum is also similar to that of Trichaptum abientinum $(40.61 \mathrm{Mb})$ belonging to the order Polyporales in phylum Basidiomycota and Melanomma pulvi-pyris $(42.09 \mathrm{Mb})$ belonging to the order Pleosporales in Phylum Ascomycota [30].

\section{Mobile elements}

A $1.68 \%$ fraction of the P. lilacinum genome was estimated to consist of repeated sequences. A total of 105 retrotransposons (class I), 2 DNA transposons (class II), and 1 unknown element (Additional file 1: Table S3) were

Table 1 Genomic features of $P$. lilacinum

\begin{tabular}{ll}
\hline Features & P. lilacinum \\
\hline Genome Size (Mb) & 40.02 \\
$\% G C$ content & $58.57 \%$ \\
Predicted Proteins & 13266 \\
Avg. Gene Density(genes/Mb) & 303 \\
Avg. Gene length (bp) & 1512 \\
Repeat Content \% & $1.68 \%$ \\
tRNAs & 91 \\
Secreted Proteins & 1276 \\
Secondary metabolites clusters & 30 (SMURF), 46 (AntiSMASH) \\
PHI genes & 1953 \\
Proteases & 480 \\
\hline
\end{tabular}


identified in the genome. The number and family wise distribution of mobile elements in P. lilacinum genome is comparable other insect pathogenic fungal species in Hypocreales [19-22].

\section{Gene Ontology (GO) based distribution of genes} Of the total predicted genes in P. lilacinum, 9800 (73\%) had Blastp hits and 8204 proteins (62\%) had InterProScan hits. Genes having InterProScan hits were investigated for distribution into functional categories based on Gene Ontology (GO). Allocation by GO domains, "Biological Process" and Molecular Function", according to generic terms at level 3 in Blast2GO is presented in Fig. 1. Major "Biological process" groups were constituted by the following GO terms: organic substance metabolic process (18\%), primary metabolic process (17\%), biosynthesis process $(9 \%)$, establishment of localization (7\%), catabolic process $(1.5 \%)$, response to stress $(1 \%)$, and pathogenesis genes $(0.1 \%)$. Distribution according to "Molecular function" largely encompassed those genes implicated in heterocyclic compound binding (15\%), ion binding (14\%), hydrolase and lyase activity $(9.5 \%)$, oxidoreductase activity (7\%), small molecule binding (7.8\%), protein binding (7.3\%), transferase activity (6\%), carbohydrate and carbohydrate derivative binding (5.6\%) and trans-membrane transporter activity $(3.1 \%)$.

\section{Phylogenomic relationships}

Neurospora crassa belongs to the order Sordariales. The orders Hypocreales and Sordariales belong to the fungal class Sordariomycetes under Ascomycetes. In our phylogenomic analysis Neurospora crassa served as an out group. Orthologous protein clusters generated by MCL clustering tool embedded in the phylogenomic pipeline Hal [31] were sieved to get non-redundant clusters. Aligned clusters were concatenated to generate superalignments that were used to build maximum likelihood phylogeny tree.

The constructed phylogenomic tree (Fig. 2) mimicked the recent taxonomic classification with the representative species belonging to the five families in Hypocreales being clustered under a separate node from Neurospora crassa. The family Nectriaceae comprising primarily of plant-pathogens such as F. graminearum and $F$. oxysporum diverged the earliest. Members of Hypocreaceae (represented by Trichoderma reesei in this analysis) are widely known to possess mycoparasitic life style. Hypocreaceae composed a sister node to Cordycipitaceae family that holds insect-pathogenic fungal species Beauveria bassiana and Cordyceps militaris. The family Clavicipitaceae, which by and large includes pathogens of insects, was represented by Metarhizium robertsii, Metarhizium acridum and a nematophagous fungus Pochonia chlamydosporia in this study. Members of Ophiocordycipitaceae included Tolypocladium inflatum and P. lilacinum. T. inflatum is a pathogen of beetle larvae and $P$. lilacinum is a nematophagous fungus. Cordycipitaceae and Ophiocordycipitaceae seemed to have diverged earlier in comparison to Clavicipitaceae in the evolutionary history. Genome sequencing data of the species belonging to the remaining two families Niessliaceae and Bionectriaceae in Hypocreales has not been released as yet in the public domain by DOE (Department of Energy, http://genome.jgipsf.org/) and therefore, not included in the analysis.

Phylogenomic analysis presented in this study was largely in agreement with typical phylogenetic studies that have sampled a large number of Hypocreales species and constructed evolutionary relationships from meta-analysis of multi-gene datasets [14, 15, 32]. However, taxonomic refinement of Clavicipitaceae, which was grouped into Clavicipitaceae A, B and C by multi-gene phylogenetic analysis, could only be achieved by phylogenomic analysis done using genome-scale data that inferred polyphyletic origin of taxons with entomopathogenic fungi in three separate families in Hypocreales [33, 34]. Our results were in absolute confirmation with a previous report that presented an elaborate account of phylogenomic relationships of Hypocreales [13]. Our phylogenomic analysis indicated lifestyle transitions among the different species in the Hypocreales from plant-pathogens in initially diverging taxon (Nectriaceae) to mycoparasitism (Hypocreaceae) and entomopathogenic trophic behaviors (Cordycipitaceae, Ophiocordycipitaceae and Clavicipitaceae).

Taxonomic placement of $P$. lilacinum remained ambiguous until recent. The fungus was previously placed under Trichocomaceae that contains the very familiar genus Penicillium. Genome-scale phylogeny constructed in this study clearly places $P$. lilacinum among taxons with insect-pathogens and under the family Ophiocordycipitaceae along with $T$. inflatum.

\section{Potential pathogenesis-related genes}

To find potential pathogenesis-related genes, a whole genome blast analysis was conducted against the pathogen-host interaction (PHI) gene database version 3.6 [35] at $\mathrm{E}<1 * 10^{-20}$. PHI db catalogs experimentally validated pathogenic, virulence and effector genes from fungal, Oomycete and bacterial pathogens of fungi, insects, plants and animals hosts. Homolog identification in PHI db, therefore, indicates towards putative role of the gene in pathogenesis. Approximately $14.7 \%$ ( $N=1953$ genes) of the protein-coding genes with Blastp hits in the genome of P. lilacinum showed homology with the genes present in the PHI $\mathrm{db}$.

Total number of genes sharing homology with PHI db in the 10 Hypocreales genomes analyzed in this study is 


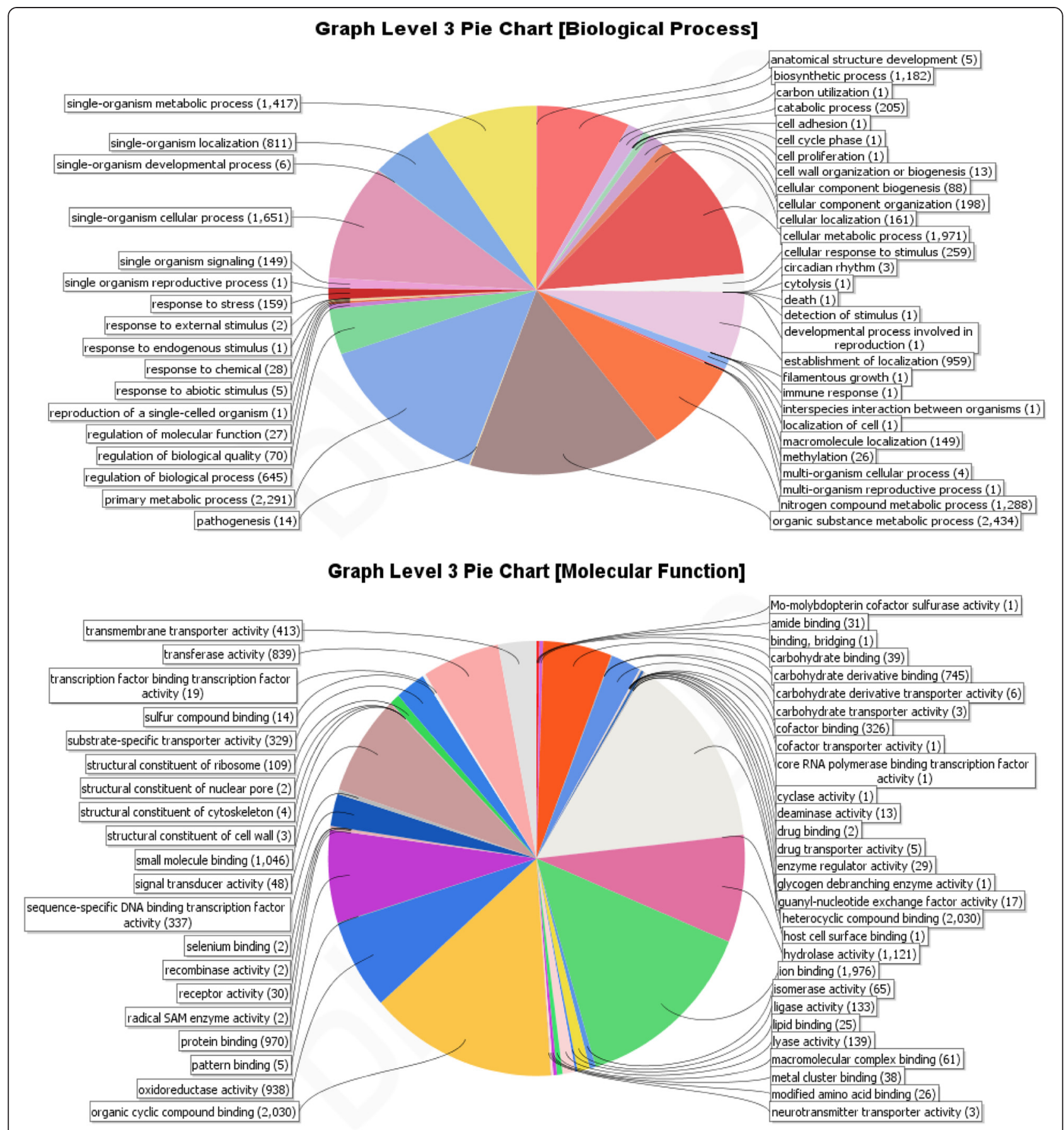

Fig. $1 \mathrm{GO}$ based functional annotation of genes present in the $P$. lilacinum genome. Biological Process domains and Molecular function domains

presented in Additional file 1: Table S4. Using Blastp $\left(\mathrm{E}<1^{*} 10^{-20}\right)$ and PHI version 3.6, highest number of PHI homologous genes were observed in plant pathogens F. oxysporum (2486 genes), which was followed by P. lilacinum (1953 genes). The insect pathogens $M$. acridum and $M$. robertsii were found to possess 1477 and 1593 genes respectively. Surprisingly, the genome of the other nematophagous fungus $P$. chlamydosporia contained only 1402 PHI homologs as compared to 1953 homologs in $P$. lilacinum.

Genes in PHI db (ver 3.6) were further sieved to select a set of genes that were experimentally validated for their role in pathogenicity by knock-out experiments. Blastp search revealed presence of 469 (3.53\%) homologs to "loss 


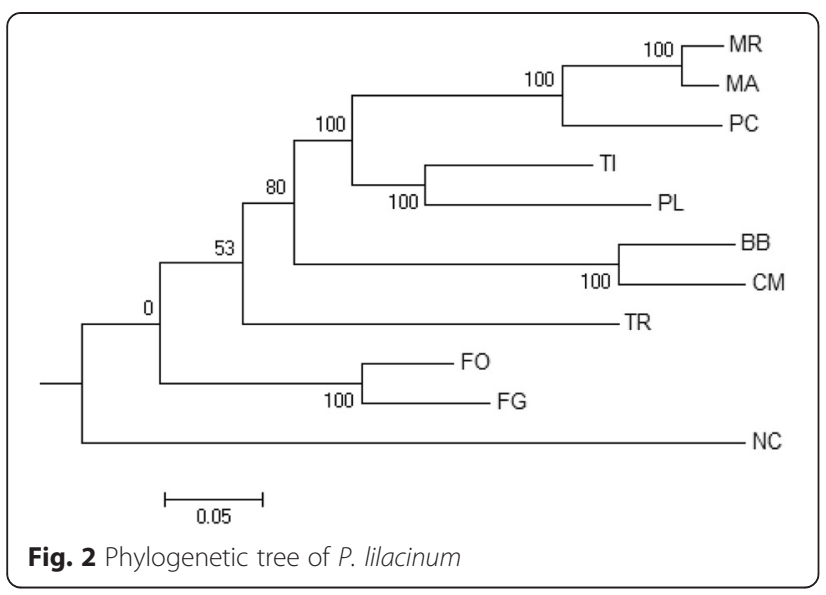

of pathogenicity genes", 92 (0.69 \%) "increased virulence genes", 1311 (9.9\%) "reduced virulence genes", 40 (0.3\%) "effector plant virulence genes", 303 (2.28 \%) "mixed pathogenesis genes" and 226 (1.70\%) "lethal genes" in the $P$. lilacinum genome.

To identify the putative involvement of the pathogenesis related genes in biological and molecular processes, Blast2GO based annotation was carried out. Based on presence of the GO domains, PHI genes were grouped into 30 biological-functional groups and 37 molecularfunction groups (Fig. 3). Major biological process groups included genes related to cellular metabolic processes (30\%), primary metabolism (34\%), biosynthetic processes (19.81\%), regulation of biological processes (14\%), establishment of localization (18\%) and response to stress $(1.5 \%)$. However, unlike $40 \mathrm{PHI}$ genes in $P$. chlamydosporia, only one PHI gene was found under GO term "pathogenesis" in P. lilacinum. Fisher exact test was done for statistical evaluation of difference in genetic make-up between $P$. lilacinum and $P$. chlamydosporia with respect to important genes. Additional file 1: Table S5 shows that significantly different proportion of establishment of localization, primary metabolic process, glycoside hydrolases, secondary metabolites, response to stress genes were present in $P$. lilacinum as compared to $P$. chlamydosporia, with abundance of serine proteases in P. lilacinum.

These findings probably indicate towards differences in the genetic arsenals between the two nematophagous fungi, $P$. lilacinum and P. chlamydosporia, belonging to two different families Ophiocordycipitaceae and Clavicipitaceae respectively of Hypocreales. Fewer matches with GO term "relationship between organisms" was not unexpected as only 14 genes in the complete genome could be identified under the category "pathogenesis" in Blast2GO based annotation of the genome (Fig. 1). Interestingly, of the 1, 259 protein-coding genes identified as associated with the GO terms "hydrolase activity" and "lyase activity" under "Molecular Function" domain in whole genome analysis (Fig. 1),
283 hydrolase and 26 lyase activity signatures were found in the PHI db, which is suggestive of their importance in pathogenesis (Fig. 3).

\section{Secreted proteins in $P$. lilacinum}

The secreted proteome plays a crucial role in defining the ability of a fungal pathogen to interact with the host and the environment. Using the online tool SignalP (ver 4.1), a total of 1,276 secreted peptides were predicted in P. lilacinum genome, of which 184 had homologs in PHI $\mathrm{db}$ (Additional file 1: Table S6). The number of secreted proteins reported for the insect pathogens $M$. robertsii and $M$. acridum [20, 21] and plant pathogens [36] and non-pathogens [37] are estimated using SignalP (ver 3) instead of the recent version SignalP (ver 4.1) and therefore, a comparison between $P$. lilacinum genome and the remaining species is difficult. Considerably large assimilation of secretary proteins in P. lilacinum genome could indicate towards increased complexity of secreted proteome for improved adaptation to environment.

\section{Signal transduction and gene regulation}

Multiple lifestyles acquired by the fungus $P$. lilacinum demands for swift adjustments to varied environments and host. Genes involved in signal transduction and gene regulation are crucial in lifestyle transitions. Fungal Gprotein coupled receptors (GPCRs) are required for transducing environmental cues, which involve niche recognition, nutrient sensing and recognition of host immune system $[34,38]$. In pathogenic fungi Parastagonospora nodorum, alpha subunits of $\mathrm{G}$ protein are found important in causing plant-disease by transducing extracellular signals [39]. In Magnaporthe sp., the Pth11-like GPCR triggers cell differentiation in response to plant inductive cues and mediates pathogenicity [40]. P. lilacinum genome encoded 33 GPCRs, compared to an average of 32 in other fungi [20]. 12 GPCRs shared homology with Pth11-like GPCRs (Additional file 1: Table S7). Number of total GPCRs and Pth11-like GPCRs in P. lilacinum genome are comparable to that of C. militaris [1] but much lesser than sequenced Clavicipitaceae fungi, Metarhizium spp. [20].

Histidine kinases (HK) are largely implicated in environment sensing and stress responses in fungi [41]. P. lilacinum possesses $24 \mathrm{HK}$ proteins compared to 17 in $M$. robertsii and 9 in $M$. acridum. Substantially increased number of HKs could indicate towards efficient signal transduction capacity of the multi-trophic fungi, P. lilacinum, under rapidly changing environmental conditions.

Protein kinases (PKs) are important in regulation of cellular and metabolic processes primarily signal transduction. 140 PK genes were identified in P. lilacinum as compared to 153 in Pochonia chlamydosporia, 167 in C. militaris, 158 in M. robertsii and 192 


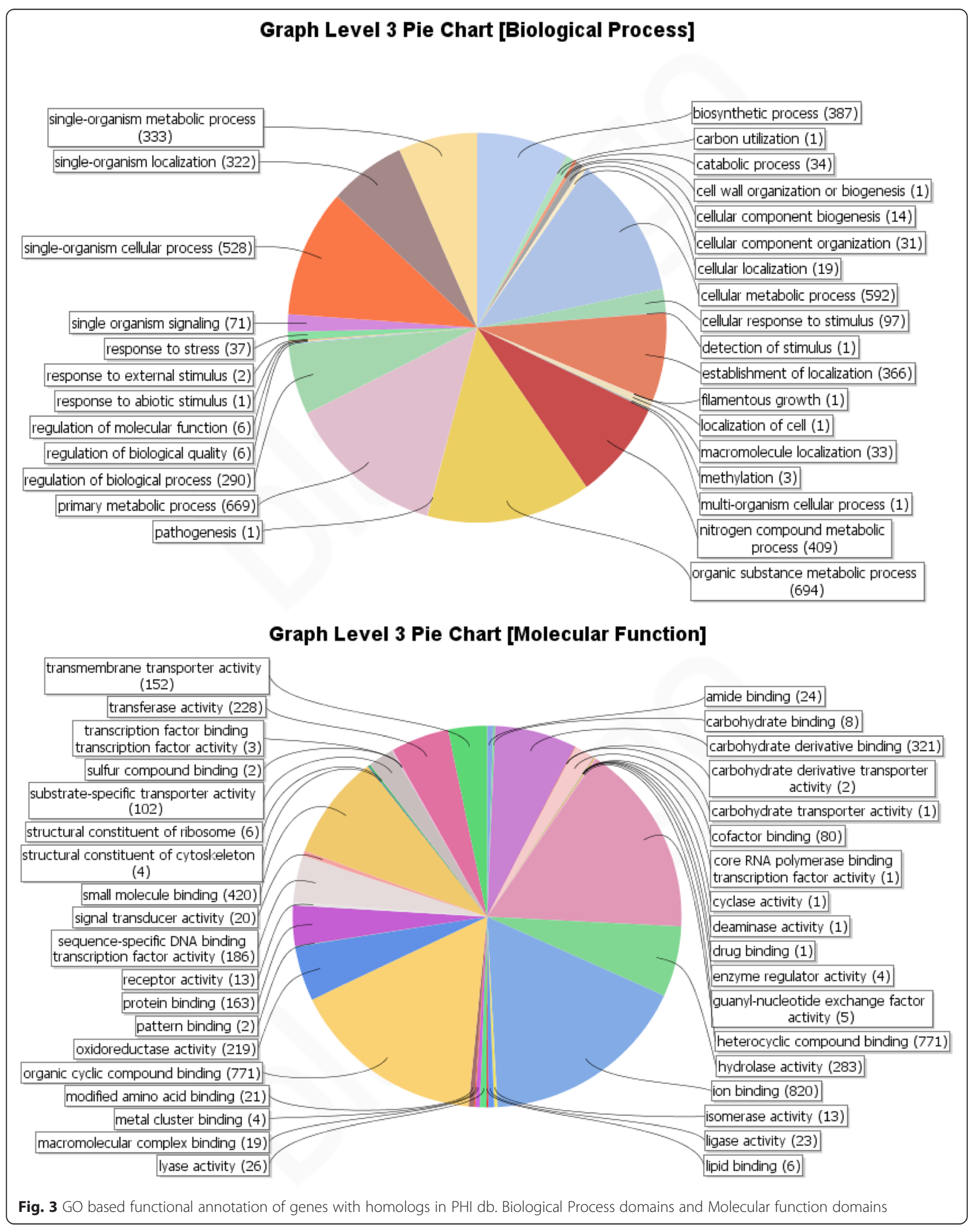


in $M$. acridum. Presence of homologs for a significant subset (116 genes) of PKs in PHI db emphasizes possibility of these genes in pathogenicity. Number of signal transduction genes and their homologs in PHI $\mathrm{db}$ has been shown in Fig. 4 .

\section{Genes involved in transportation and detoxification of compounds}

In pathogenic and parasitic fungi, ATP-binding cassette $(\mathrm{ABC})$ transporters are largely implicated in defense mechanism and protect the fungus from secondary metabolites or toxins produced by the host [42]. The $P$. lilacinum genome coded for $71 \mathrm{ABC}$ transporters, of which 61 had homologous gene present in the PHI $\mathrm{db}$. Another important family of transporters known as major facilitator super-family (MFS) is frequently involved in the transport of a wide range of substrates and nutrient sensing [42]. The genome of $P$. lilacinum encoded 266 MFS transporters, of which 176 genes had homologs in PHI db.

Cytochrome P450 (CYP) monooxygenase superfamily mediates numerous functions in fungi. Most importantly, CYPs contribute in multifaceted metabolism and adaptation to varied ecological niches [43]. Filamentous fungi produce an array of secondary metabolites, biosynthesis of many being mediated by CYPs [44, 45]. CYPs are also known to contribute towards pathogenicity and life style of fungi. In P. lilacinum 100 CYP genes were identified, of which only 45 exhibited homologous counterparts in the PHI db. Number of CYP enzymes in $P$. lilacinum is similar to Clavicipitaceae fungi ( $P$. chlamydosporia, $M$. robertsii and $M$. acridum) but is more than previously reported plant pathogenic (F. graminearum, M. oryzae) fungi. Additional file 1: Table S8 enlists Pth11 like GPCRs, Histidine kinases, Protein kinases, ABC, MFS and CYP genes in P. lilacinum. Number of transportation \& detoxification genes and their homologs in PHI base has been shown in Fig. 4.

\section{Hydrolytic enzymes}

To be a successful parasite, saprophyte and endophyte, $P$. lilacinum codes for an array of hydrolytic enzymes including proteases, chitinases, lipases and esterases. The size of hydrolytic enzyme families with putative biological function and number of homologs in PHI db has been presented in Fig. 4. Our analysis of the P. lilacinum genome unraveled the likely genetic requirements to invade the egg shells of nematodes by disintegration of egg shell components.

\section{Proteases}

By carrying out a Blastp (batch Blast) search against the MEROPS protease database [46, 47], we identified 480 genes coding for proteases in the $P$. lilacinum genome. According to their catalytic type these proteases were divided into five categories, which were further distributed into 72 families (Additional file 1: Table S9). Similar to other entomopathogenic fungi, serine proteases with 209 genes constituted the largest category of proteases in P. lilacinum, the three major classes being prolyl oligopeptidases (S09; 69 proteins), prolyl aminopeptidases (S33; 53 proteins) and subtilisins (S08; 40 proteins, S53; 9 proteins). Subtilisin-like serine proteases produced by $P$. lilacinum have been identified to degrade protein components of both nematode and insect eggs [48]. In addition, reports indicate that subtilisin-like serine proteases could have been crucial during the evolution of pathogenicity of nematode-trapping fungi against nematodes [48]. In nematophagous fungus Pochonia chlamydosporia, in addition to subtilisins, serine carboxypeptidases are also instrumental in the penetration of nematode egg

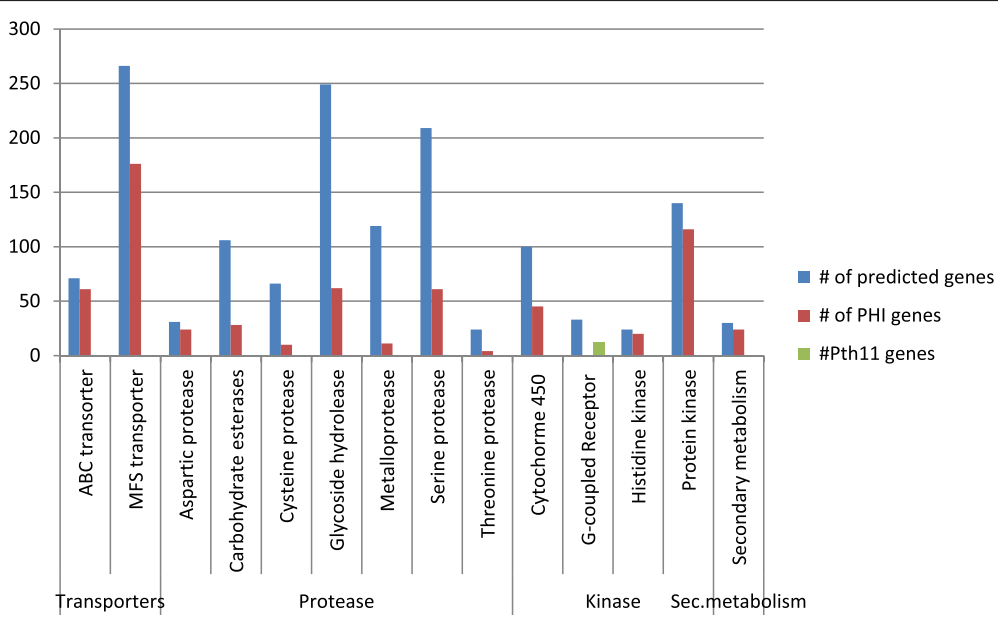

Fig. 4 Gene families important in nematode pathogenesis and their homologs in PHI db 


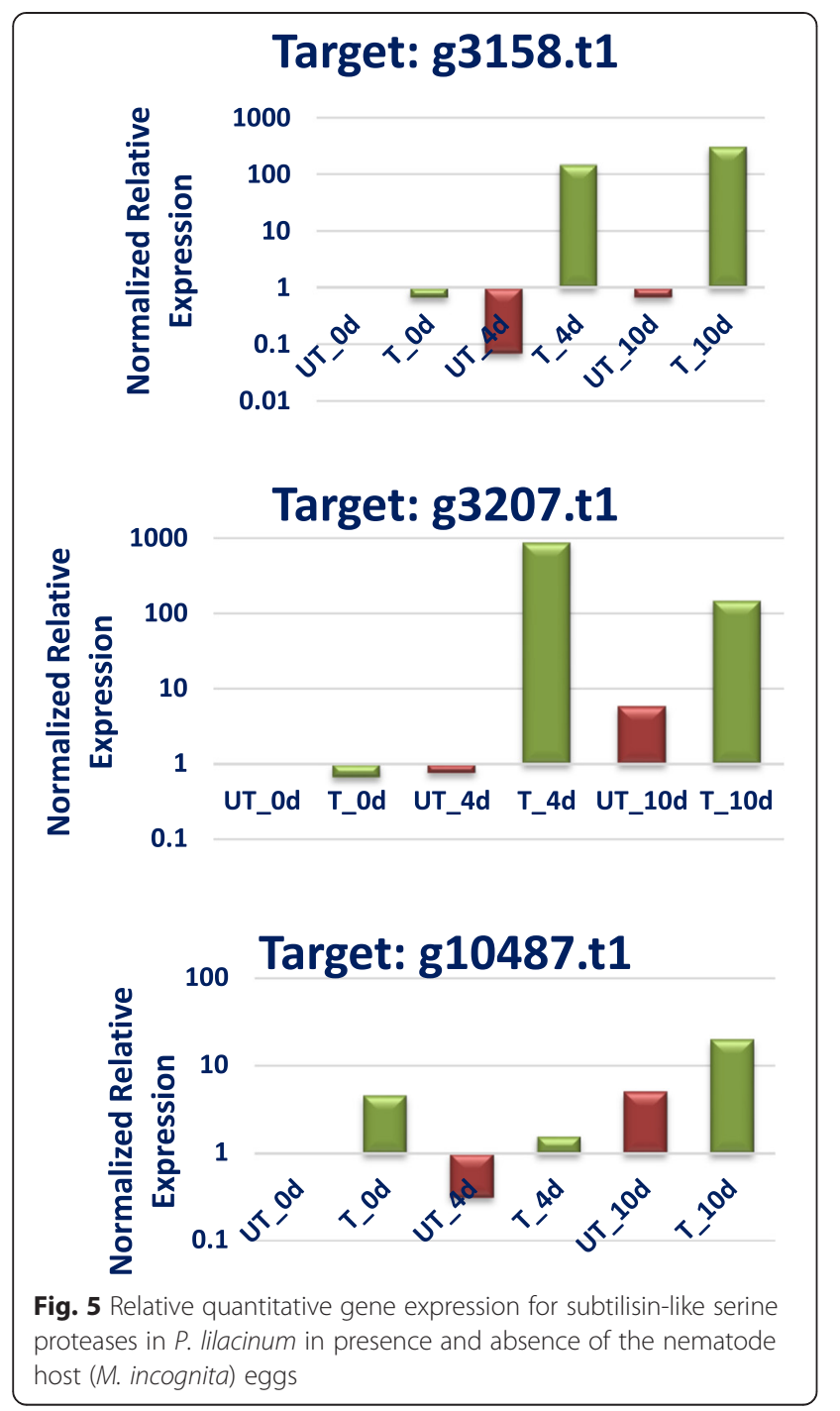

shell [49]. $59 \%$ of serine proteases showed expression under endophytism in P. chlamydosporia [22]. The $P$. lilacinum genome coded for 35 serine proteases of S8 family (subtilisins), 9 serine proteases of S53 (subtilisins) and 11 serine proteases of S10 family (serine carboxypeptidases). Expression of $50 \%$ of genes belonging to S10 family during root endophytism in Pochonia chlamydosporia, underscored the importance of serine carboxypeptidases in disintegration of the nematode egg shell. In our study, we found that 61 genes coding for serine proteases had homologous present in the PHI db (Additional file 1: Table S10), of which 15 were subtilisins (S08) and 9 were serine carboxypeptidases(S10), which further supported their role in pathogenicity. Similar to $P$. chlamydosporia, the second largest group of proteases in P. lilacinum genome was made of Metalloproteases, with 119 genes spread over 25 families. The largest family within Metalloproteases was glutamate carboxypeptidases
(M20; 18 genes). The important classes of metalloproteases that were reportedly expressed during endophytism in P. chlamydosporia were fungalysins (M36), carboxypeptidases (M28) and deuterolysins (M35). The $P$. lilacinum genome harbored 2 fungalysins, 11 carboxypeptidases and 7 deuterolysins quite in an adequate proportion with Pochonia chlamydosporia genome. Remaining proteases in the genome belonged to threonine protease class with 24 genes, cysteine proteases with 66 genes and aspartic proteases with 31 genes.

\section{Glycosides hydrolases and carbohydrate esterases}

The egg-shell components of nematodes include structural polysaccharides such as cellulose, chitin and mannan. In order to penetrate the nematode egg, $P$. lilacinum needs to produce glycoside hydrolases $(\mathrm{GH})$ and carbohydrate esterases (CE) for degradation of polysaccharides. Using CAZy database [50] and performing HMMER [51] scan based on the profile compiled in dbCAN release 2.0; we predicted presence of 249 glycoside hydrolases $(\mathrm{GH})$ and 106 carbohydrate esterases (CE) in P. lilacinum genome. The size of the GH family in $P$. lilacinum is very close to $F$. graminearum (269 genes) of Nectriaceae and P. chlamydosporia (281 genes) of Clavicipitaceae, as compared to small sized family in M. acridum (176 genes) and M. robertsii (194 genes) of Clavicipitaceae (Additional file 1: Table S11). Chitinases coded by 28 genes of GH18 class were the most abundant of all glycoside hydrolases in P. lilacinum. 13 genes encoding for cellulases belonging to GH5-GH12 families were identified in this genome. Cellulases were found to be expressed during root colonization in $P$. chlamydosporia and thus could be important in life style transitions in P. lilacinum.

Carbohydrate esterases (CEs) carry out the de-O or de-N-acylation of esters or amides and other substituted saccharides, in which sugars play the role of alcohol and amine. Our analysis showed that Hypocreales fungi (P. lilacinum, $P$. chlamydosporia, $F$. graminearum and Metarhizium spp.) have genes from 15 of the $16 \mathrm{CE}$ families, with CE15 being the only missing family (Additional file 1: Table S12). We identified 106 genes encoding carbohydrate esterase enzymes in P. lilacinum, with CE10 being the largest family. Members of families CE1 and CE10 possessed maximum number of genes coding for carbohydrate esterases. CE1 and CE10 share the common activities of carboxylesterase and endo-1,4- $\beta$-xylanase [52]. However, they greatly differ in substrate specificity. The number of CE enzymes in $P$. lilacinum genome was more than that reported for Clavicipitaceae family and less than Nectriaceae family under the Hypocreales order. 
In P. lilacinum $62 \mathrm{GH}$ and $28 \mathrm{CE}$ genes had homologs counterparts in PHI db (Additional file 1: Table S13). The genome seems to be enriched with hydrolytic enzymes and employs an assortment of proteases, glycoside hydrolases, and carbohydrate esterases to breach the egg-shell barrier in order to parasitize nematode.

\section{Secondary metabolite genes and clusters}

Species in Hypocreales are known for production of secondary metabolites that include toxins and pharmaceutically active components. $P$. lilacinum is widely known to produce a leucinostatin named as paecilotoxin. Leucinostatins are linear nine residue peptabiotics, characterized by $\mathrm{N}$-terminal proline acetylated by MeHA and C-terminal B-Ala amidated by DPD. Leucinostatin is ubiquitously present among all the $P$. lilacinum isolates and its production does not correlate with the habitat or host of the different strains [53]. Production of secondary metabolite other than paecilotoxin has not been reported to date from $P$. lilacinum. Genetic analysis to unveil gene clusters for production of different types of secondary metabolites would help in identification of important bioactives.

The genes responsible for biosynthesis, export, and transcriptional regulation of secondary metabolites are often found in contiguous gene cluster. Secondary metabolite unique regions finder (SMURF) is a web-based tool [54] that finds secondary metabolite biosynthesis gene clusters and pathways in fungal genomes based on their genomic context and domain content. Another in silico pipeline antiSMASH 2.0 [55], identifies bio-synthetic loci coding for secondary metabolites including oligosaccharide antibiotics, phenazines, homoserine lactones, thiopeptides, furans and phosphonates, in addition to secondary metabolite classes: polyketides, non-ribosomal peptides, terpenes, aminoglycosides, aminocoumarins, indolocarbozoles, lantibiotics, bacteriocins, nucleosides, beta-lactams, butyrolactones, siderophores, melanins. Using SMURF, 30 secondary metabolite genes were identified (Additional file 1: Table S14). These genes included 12 PKS (polyketide synthase), 2 PKS-like enzymes, 7 NRPS (nonribosomal polyketide synthase), 7 NRPS-like enzymes, 1 hybrid and 1 DMAT (dimethyllacetyltransferase). In comparison to other sequenced fungi in Hypocreales, SMURF analysis indicated that $P$. lilacinum has lesser capacity for the production of bio-actives and toxins. However, antiSMASH run on the genome data identified 46 clusters (10 nrps, 16 type 1 pks, 9 terpene, 1 lantipeptide, 1 linaridin, and 9 other secondary metabolites clusters), which is close to the maximum (51) reported in entomopathogenic fungus M. robertsii $[20,21]$. AntiSMASH based Functional annotations of the 46 clusters are presented in (Additional file 1: Table S15). AntiSMASH analysis suggested that $P$. lilacinum could encode several non-conventional secondary metabolites by using alternate gene clusters.

Bioinformatics tool Natural Product Domain Seeker (NaPDoS) (http://napdos.ucsd.edu) (Db version: pksdb_20111206) was used to detect and extract condensation (C) and ketosythase (KS) domains through a broad set of manually curated reference genes from well-characterized chemical pathways. We found 18 PKS-derived KS domains and 10 NRPS-derived C-domain in P. lilacinum (Additional file 1: Table S16).

\section{Quantitative real-time PCR}

To validate importance of serine proteases expressed by P. lilacinum in nematode pathogenesis, quantitative realtime PCR analyses were carried out. Three subtilisin-like serine proteases, namely, g3158.t1, g3207.t1, and g10487.t1, were selected as target genes for the analyses and actin gene was chosen as reference gene. Real-time PCR results confirmed up-regulation of all three subtilisin-like serine proteases and showed that, in the presence of eggs of nematode host (Meloidogyne incognita), the copy number of the target genes of $P$. lilacinum was increased in comparison to control (fungus grown in absence of the host) Fig. 5. Notably, relative expression of g3158.t1, g3207.t1 genes was significantly higher (with expression increasing from 148.0561 to 885.2861 -folds) on 4th and 10 th day post inoculation of $P$. lilacinum with the host nematode eggs. The outcome of quantitative real-time PCR analyses underscore potential role of these subtilisin-like serine proteases in nematode egg shell degradation.

\section{Conclusions}

The whole genome annotation and comparative genomic analyses of the first draft genome sequence of monophyletic genus Purpureocillium lilacinum provided insight into its genetic make-up and phylogenetic placement. Presence of a variety of hydrolytic proteins in the genome illustrated putative genetic machinery involved in nematophagous activity. Quantitative real-time PCR analyses corroborated role of serine proteases in nematode pathogenesis. Identification of a plethora of GPCRs, transporter proteins, CYPs and protein and histidine kinase proteins indicate the tentative genetic components important for life style transitions and substantiate the observed multi-trophic behaviour viz., saprophyte, egg-parasite, and plant-endophyte of $P$. lilacinum. Sizable representation of important enzyme families in pathogen-host interaction database corroborates importance of the identified proteins in nematode pathogenesis. Significantly large number of secondary metabolite clusters identified through antiSMASH underscores immense capacity of $P$. lilacinum genome to synthesize a variety of typical and atypical secondary metabolites. The gene lists for various protein families provided here, would be useful for 
future experiments. In addition, phylogenomic study involving nine other fungi from five different families under the order Hypocreales, provide confirmation that $P$. lilacinum, which was earlier erroneously grouped with Paecilomyces under the order Eurotiales, belongs to the Ophiocordycipitaceae family in the order Hypocreales. In conclusion, findings of this genomic analysis would be beneficial for development of effective biological-control approaches to manage agricultural damage incurred by plant parasitic nematodes.

\section{Methods}

\section{Fungal strain}

The details of $P$. lilacinum strain used in the study has been studied in the laboratory for more than 7 years [56]. P. lilacinum grown on potato dextrose agar (PDA) at $25{ }^{\circ} \mathrm{C}$ for 8 days was inoculated in potato dextrose broth (PDB) and incubated at $25{ }^{\circ} \mathrm{C}$ for 5 days with shaking at $120 \mathrm{rpm}$. Genomic DNA was isolated using the DNeasy Plant Maxi Kit (Qiagen) following the fungal DNA isolation protocol. Total DNA obtained was subjected to quality control by agarose gel electrophoresis and quantified by the nanodrop method.

\section{Genome sequencing and assembly}

Genomic sequencing was performed at MOgene LC, USA, using next generation sequencing technology Illumina. Two paired end libraries (insert sizes $180 \mathrm{bp}$ and $500 \mathrm{bp}$ ) and one mate pair library (5Kb) were constructed. Briefly, for paired end libraries, $1 \mu \mathrm{g}$ genomic DNA in total volume of $50 \mu \mathrm{L}$ was fragmented using Covaris to generate dsDNA fragments with 3' or 5' overhangs. After shearing, the ends of DNA fragments were repaired by adding $40 \mu \mathrm{L}$ of Illumina end repair mix and incubation at $30{ }^{\circ} \mathrm{C}$ for $30 \mathrm{~min}$, followed by adenylation of 3 ' ends (added 2.5uL of A-Tailing mix and incubated at $37^{\circ} \mathrm{C}$ for $30 \mathrm{~min}$ ). Double-stranded Illumina paired-end adapters were ligated to the polyadenylated DNA. Ligated product was purified by loading $20 \mu \mathrm{L}$ of samples on $1 \%$ agarose gel. For $180 \mathrm{bp}$ insert size, bands from the gel ranging from 300-400 bp were excised and for $500 \mathrm{bp}$ insert size, bands ranging between 620-700 bp were excised. Excised bands were then purified using QIA gel extraction kit. $20 \mu \mathrm{L}$ of purified sample were PCR amplified using the following program $\left(98^{\circ} \mathrm{C}\right.$ for $30 \mathrm{~s} ; 10$ cycles of: $98{ }^{\circ} \mathrm{C}$ for $10 \mathrm{~s}, 60{ }^{\circ} \mathrm{C}$ for $30 \mathrm{~s}, 72{ }^{\circ} \mathrm{C}$ for $30 \mathrm{~s} ; 72{ }^{\circ} \mathrm{C}$ for $5 \mathrm{~min}$ ). DNA libraries were purified using AMPure XP beads. KAPA was done to quantify the libraries, which were then normalized and pooled at 4nM concentration.

For constructing mate pair library of insert size $\sim 5 \mathrm{~Kb}$, $4 \mu \mathrm{g}$ of gDNA was tagmented using mate pair tagment enzyme followed by strand displacement at $20{ }^{\circ} \mathrm{C}$ for 30 min. AMPure beads were used to purify strand displaced samples. Smaller fragments of size less than
1500 bp were removed. $30 \mu \mathrm{L}$ of samples were run on $1 \%$ agarose gel and bands ranging from 5-7 kb were excised and cleaned using Zymoclean large fragment DNA recovery kit. Purified fragments were circularized with an overnight incubation at $30{ }^{\circ} \mathrm{C}$ followed by DNA exonuclease treatment to remove any linear molecules still remaining in the circularization reaction. Circularized DNA molecules were then sheared to small sized fragments (approximately 300-1000 bp). After shearing, the ends of DNA fragments were repaired, ligated with appropriate adaptor, PCR amplified, and purified in the manner described above for paired end library preparation.

Libraries were quantified and loaded into Illumina flow-cells at concentrations of 1.4-1.75 pM (HiSeq 2000). Cluster generation was performed in a cBOT automated cluster generation system. Base calling was done using Illumina pipeline software. A total of $5.4 \mathrm{~Gb}$ of pair-end (180 bp and $500 \mathrm{bp}$ inserts) and $2.6 \mathrm{~Gb}$ of mate-pairs ( $5 \mathrm{~kb}$ inserts), were produced. A total of 8 Gb raw data was subjected to adaptor- and qualitybased trimming. Quality-passed data was assembled using the de novo genome assembler AllpathsLG [57]. Reads with overlaps were first combined to form contigs. The reads were mapped back to contigs. With paired-end reads, contigs from the same transcript, as well as the distances between these contigs, were detected. In order to generate scaffolds, contigs were connected using " $\mathrm{N}$ " to represent unknown sequences between two contigs. Mate-pair reads were used for gap filling of scaffolds in order to get sequences with minimal N's and the longest length. By mapping the reads to contigs, the genome sequence was assembled into 301 scaffolds with total gap size of 526,913 bases. The whole genome project has been deposited at https:// submit.ncbi.nlm.nih.gov/subs/wgs/ under Bioproject number PRJNA284314.

\section{Gene prediction, annotation, protein classification and phylogenomic analysis Genomic structural annotation}

Maker [25] was used to structurally annotate the genomic assembly. EST data for 21 different fungal species, and protein sequences from 10 different fungal species were used to inform the annotation processes. SNAP [58], GeneMarkHMM [59], and Augustus [60], each trained on fungal data and informed with EST and protein sequences as described above, were used to predict genes.

\section{Functional annotation}

Repeat elements in the genome were analyzed by RMBlastN search against the RepeatMasker library (open 3.2.9) [61]. The DNA transposases and reterotransposaes 
were annotated by blastp searches against Repbase (repeatmaskerlibraries-20140131) [62]. To estimate genome size k-mer analysis was carried out using KmerGenie v1.6976 [63]. Pseudogene identification was conducted using the PseudoPipe tool [64]. tRNAs were identified using tRNA scan-SE [28, 29]. Protein set derived from genomic structural annotation was annotated using Blastp versus the NCBI/Genbank NR protein database [65], and using the protein domain/motif prediction program InterProScan [66]. Genes were also annotated by using Blast2GO [67] based on the terms "biological function" and "molecular process" in Gene Ontology (GO). SignalP [68] was used to identify putative secreted proteins. Proteins coding for proteases were classified by conducting Blastp (batch) against the MEROPS database $[46,47]$. In order to carry out classification of carbohydrate-active enzymes (CAZymes), a HMMER scan was carried out based on the profiles compiled in dbCAN release 2.0 on the CAZy database. To identify G-protein-coupled receptors in the genome, local BLAST to GPCRDB sequences [69] was carried out and those proteins containing seven transmembrane helices were selected using TMHMM [70]. Homologs of the Pth11-like GPCRs were confirmed by local Blastp analysis (E value of 1e-20). Secondary metabolite genes were predicted by SMURF [54]. AntiSMASH [55] analysis on the whole genome sequence was performed to identify classical and novel clusters coding for secondary metabolites. Blastp search (E value $<1^{*} 10^{-20}$ ) against pathogen-host interaction (PHI) database ver 3.6 [35] was carried out to identify putative pathogenesis genes.

\section{Phylogenomic analysis}

Whole genome nucleotide, gene and protein sequences of nine species belonging to the fungal order Hypocreales namely, Fusarium oxysporum (http://www.ncbi.nlm.nih.gov/bioproject/18813), Fusarium graminearum (http:// www.ncbi.nlm.nih.gov/bioproject/PRJNA235346), Trichoder ma reesei (http://www.ncbi.nlm.nih.gov/bioproject/PRJNA2 66930), Beauveria bassiana (http://www.ncbi.nlm.nih.gov/ bioproject/38719), Cordyceps militaris (http://www.ncbi.nlm. nih.gov/bioproject/PRJNA41129/), Metarhizium robertsii (http://www.ncbi.nlm.nih.gov/bioproject/PRJNA245140/), Metarhizium acridum (http://www.ncbi.nlm.nih.gov/bioproject/38715/), Pochonia chlamydosporia (http://www.ncbi. nlm.nih.gov/bioproject/68669/) and Tolypocladium inflatum (http://www.ncbi.nlm.nih.gov/bioproject/PRJNA73163/), were downloaded from NCBI database (http://www.ncbi. nlm.nih.gov/). In the absence of gene/protein sequences of Pochonia chlamydosporia and Tolypocladium inflatum, structural annotation (prediction of gene and protein sequences) of their respective BioProjects present in NCBI was carried out using Augustus [60]. To serve as an out group in the phylogenomic analysis, protein sequence of
Neurospora crassa was downloaded (http://www.broadinstitute.org/annotation/genome/neurospora/). Hal, an automated pipeline for phylogenetic analysis of genome-scale data [31] was used for multiple alignment and generation of orthologous protein clusters [http://sourceforge.net/projects/bio-hal/]. Orthologous protein clusters [71] were identified by using Blastp and Markov Cluster Algorithm (MCL) tool [72] integrated in Hal pipeline. Only nonredundant clusters that consisted of protein sequences with best hits to proteins from own cluster were filtered out. Protein sequences for these orthologous clusters were extracted from proteome dataset and aligned using MUSCLE [73]. Gblocks was used to remove poorly aligned positions and highly divergent sequences [74]. Individual alignments were parsed by minimum alignment length and concatenated using FasConcat [75] for generation of super alignments. RAxML [76] with PROTCAT setting, Dayhoff amino acid substitution model and 1000 bootstrap replications was run on super-aligned sequences for construction of phylogenetic tree.

\section{Quantitative real-time PCR \\ Total RNA extraction and CDNA preparation}

The $P$. lilacinum strain used in the study was grown on potato dextrose media in the presence of the nematode Meloidogyne incognita eggs. Same strain grown in the absence of Meloidogyne incognita eggs served as control. The cultures were incubated for $0,4,7$ and 10 day/s before RNA isolation. Total RNA was extracted using PureLink RNA Mini Kit (Invitrogen, USA). cDNA for all the samples corresponding to different time points $(0,4,7$ and 10 day/s) was prepared using iScript cDNA synthesis kit (Bio-Rad Laboratories, Inc.) using following incubation conditions: $5 \mathrm{~min}$ at $25^{\circ} \mathrm{C}, 30 \mathrm{~min}$ at $42{ }^{\circ} \mathrm{C}$ and finally $5 \mathrm{~min}$ at $85^{\circ} \mathrm{C}$. Quantification of RNA and cDNA samples was done using the H1M microplate reader (Biotek, USA).

\section{Primer designing and real-time PCR analysis}

To facilitate the real-time PCR analysis of the three serine protease genes and reference actin gene under same reaction conditions, primers were designed using Primer Express 3.0 software (PE Applied Biosystems, USA) under default parameters. The forward and reverse primers, wherever possible, were designed spanning an intron to detect any genomic DNA contamination. The primer sequences are given in Additional file 1: Table S17.

\section{Gene expression and relative quantification}

Quantification of gene expression was performed on CFX- 96 real-time PCR detection system (Bio-Rad Labs, Inc.). The PCR mixture contained 100 ng of total cDNA template, $10 \mu \mathrm{l}$ of $2 \mathrm{X}$ Sso Fast Eva Green Supermix Dye (Bio-Rad Labs, Inc.), $100 \mathrm{nM}$ forward and reverse primer in a final volume of $20 \mu \mathrm{l}$. Thermal cycling conditions 
were as follows: $30 \mathrm{~s}$ at $95{ }^{\circ} \mathrm{C}$ followed by 40 repeats of $10 \mathrm{~s}$ at $95{ }^{\circ} \mathrm{C}, 30 \mathrm{~s}$ at $60{ }^{\circ} \mathrm{C}$ and a melt curve from $65{ }^{\circ} \mathrm{C}$ to $95{ }^{\circ} \mathrm{C}$. Data collection was performed during each cycle. Relative gene expression by qPCR was performed using Actin as the reference gene for normalization of expression of target genes. PCRs with no template controls (NTC) were also performed for each primer pair. The specificity of amplicons was verified by melting curve analysis $\left(60{ }^{\circ} \mathrm{C}\right.$ to $\left.95{ }^{\circ} \mathrm{C}\right)$ after 40 cycles. Two biological replicates for each sample were used for real-time PCR analysis and three technical replicates were analyzed for each biological replicate.

\section{Statistical analyses}

Data for $\mathrm{qPCR}$ reactions were reported with standard error from the mean. The data was analyzed using the nonparametric Kruskale-Wallis test. These were then subjected to pairwise comparison. The significance level used for analysis was $P<0.05$ for all statistical tests. Statistical analyses were performed using SPSS version 12.0.

\section{Additional file}

Additional file 1: Table S1. Statistics of the completeness of the genome based on 248 CEGs. Table S2. Genome size and the number of protein-coding genes in P. lilacinum and the other Hypocreales fungi. Table S3. Repeat elements in the draft genome sequence of $P$. lilacinum. Table S4. Total number of genes sharing homology with $\mathrm{PHI} d b$ in the 10 Hypocreales genomes. Table S5. Fisher's exact test for evaluation of difference in genetic make-up between $P$. lilacinum and $P$. chlamydosporia. Table S6. Gene coding for secreted proteins sharing homology with PHI db. Table S7. GPCRs sharing homology with Pth11-like GPCRs in P. lilacinum. Table S8. Genes coding for GPCRs, Histidine kinases, protein kinases, ABC transporters, MFS and CYP450 proteins in P. lilacinum. Table S9. Genes coding for proteases in the P. lilacinum genome. Table S10. Gene coding for serine proteases with homologs in the PHI db. Table S11. Carbohydrate-degrading enzymes arranged by GH family. Table S12. Carbohydrate esterases arranged by CE family. Table S13. Glycosides hydrolase and carbohydrate esterase genes sharing homology with PHI db. Table S14. Secondary metabolites genes and clusters identified by SMURF. Table S15. AntiSMASH based functional annotation of secondary metabolite clusters. Table S16. NaPDoS analysis to detect condensation (C) and ketosynthase (KS) domains. TableS 17. Primer sequences for quantitative real-time PCR analyses. Figure $\mathbf{S} 1$. $k$-mer analysis to predict assembly size of P. lilacinum. (ZIP $201 \mathrm{~kb}$ )

\section{Abbreviations}

P. lilacinum: Purpureocillium lilacinum; PHI: Pathogen-host interaction; CAZy: Carbohydrate-active enzyme; GH: Glycoside hydrolase; CE: Carbohydrate esterase; PKS: Polyketide synthase; NRPS: Non-ribosomal peptide synthetase; MFS: Major facilitator superfamily; ABC: ATP-binding cassette; CYP: Cytochrome P450; GPCR: G-protein coupled receptor.

\section{Competing interests}

The authors declare that they have no competing interests.

\section{Authors' contributions}

All authors have read and approved the final Ms. PP was the principal Computational Genomics Scientist and coordinator of the project, involved in conceptualization of the project, study design, bio-informatics analyses, data compilation, Ms. writing, critical inputs and finalization of the manuscript; DV was involved in bio-informatics analysis, AA was involved in critical inputs and finalization of the manuscript.

\section{Acknowledgements}

This work was supported by the Biotechnology and Management of Bioresources Division, The Energy and Resources Institute, New Delhi. Sequencing was performed by the MoGene, LC, USA. The authors thank Dr Paritosh Kumar, University of Delhi South Campus and Dr Surojit Bose, Leadlnvent Technologies, India, for helping in software installations on High-Performance Computing Cluster; Dr. Mandira Kochar and Ms. Vatsala Koul, TERI Deakin Nanobiotechnology Centre, TERI, for helping in RT-qPCR analysis; Dr. Sharad Mohan, Department of Nematology, Indian Agriculture research Institute, for kindly providing $M$. incognita eggs for RT-qPCR experiments.

Received: 30 June 2015 Accepted: 18 November 2015

Published online: 25 November 2015

\section{References}

1. Zheng P, Xia Y, Xiao G, Xiong C, Hu X, Zhang S, et al. Genome sequence of the insect pathogenic fungus Cordyceps militaris, a valued traditional Chinese medicine. Genome Biol. 2011;12(11):R116.

2. Timper P, Parajuli G. Suppression of meloidogyne incognita by paecilomyces lilacinus is enhanced by planting cover crops. J Nematol. 2012:44:494-5.

3. Siddiqui ZA, Mahmood I. Biological control of plant parasitic nematodes by fungi: a review. Bioresour Technol. 1996;58:229-39.

4. Royal Society Report. Reaping the Benefits: Science and the Sustainable Intensification of Global Agriculture. London: The Royal Society; 2009.

5. Pennisi E. Armed and dangerous. Sci. 2010;327(5967):804-5.

6. Skamnioti P, Gurr SJ. Against the grain: safeguarding rice from rice blast disease. Trends Biotechnol. 2009;27(3):141-50.

7. Gurr S, Samalova M, Fisher M. The rise and rise of emerging infectious fungi challenges food security and ecosystem health. Fungal Biol Rev. 2011;25:181-8.

8. Atkinson HJ, Urwin EP, Hansen E, McPherson MJ. Designs for engineered resistance to root-parasitic nematodes. Trends Biotechnol. 1995;13:369-74.

9. Bent E, Loffredo A, McKenry MV, Becker JO, Borneman J. Detection and Investigation of Soil Biological Activity against Meloidogyne incognita. J Nematol. 2008:40(2):109-18.

10. Lamovsekl J, Urek G, Trdan S. Biological Control of Root-Knot Nematodes (Meloidogyne spp.): Microbes against the Pests. Acta agriculturae Slovenica. 2013;101-2:263-75.

11. Schapovaloff ME, Alves LF, Fanti AL, Alzogaray RA, López Lastra CC. Susceptibility of adults of the cerambycid beetle Hedypathes betulinus to the entomopathogenic fungi Beauveria bassiana, Metarhizium anisopliae, and Purpureocillium lilacinum. J Insect Sci. 2014;14:127.

12. Castillo Lopez D, Zhu-Salzman K, Ek-Ramos MJ, Sword GA. The entomopathogenic fungal endophytes Purpureocillium lilacinum (formerly Paecilomyces lilacinus) and Beauveria bassiana negatively affect cotton aphid reproduction under both greenhouse and field conditions. PLoS One. 2014;9(8):e103891.

13. Bushley KE, Raja R, Jaiswal P, Cumbie JS, Nonogaki M, Boyd AE, et al. The genome of Tolypocladium inflatum: evolution, organization, and expression of the cyclosporin biosynthetic gene cluster. PLoS Genet. 2013;9(6): e1003496.

14. Sung GH, Poinar Jr GO, Spatafora JW. The oldest fossil evidence of animal parasitism by fungi supports a Cretaceous diversification of fungalarthropod symbioses. Mol Phylogenet Evol. 2008;49(2):495-502.

15. Spatafora JW, Sung GH, Sung JM, Hywel-Jones NL, White Jr JF. Phylogenetic evidence for an animal pathogen origin of ergot and the grass endophytes. Mol Ecol. 2007;16(8):1701-11.

16. Cavello IA, Hours RA, Cavalitto SF. Bioprocessing of "Hair Waste" by Paecilomyces lilacinus as a Source of a Bleach-Stable, Alkaline, and thermostable Keratinase with Potential Application as a Laundry Detergent Additive: Characterization and Wash Performance Analysis. Biotechnol Res Int. 2012;2012:369308.

17. Khan A, Williams KL, Nevalainen HKM. Infection of plant-parasitic nematodes by Paecilomyces lilacinus and Monacrosporium lysipagum. Biocontrol. 2006B; 51: 659-678

18. Crow WT. Effects of a Commercial Formulation of Paecilomyces lilacinus Strain 251 on Overseeded Bermudagrass Infested with Belonolaimus longicaudatus. J Nematol. 2013;45(3):223-7. 
19. Martinez D, Berka RM, Henrissat B, Saloheimo M, Arvas M, Baker SE, et al. Genome sequencing and analysis of the biomass-degrading fungus Trichoderma reesei (syn. Hypocrea jecorina). Nat Biotechnol. 2008;26((5):553-60

20. Gao Q, Jin K, Ying SH, Zhang Y, Xiao G, Shang Y, et al. Genome sequencing and comparative transcriptomics of the model entomopathogenic fungi Metarhizium anisopliae and M. acridum. PLoS Genet. 2011;7(1):e1001264.

21. Hu X, Xiao G, Zheng P, Shang Y, Su Y, Zhang X, et al. Trajectory and genomic determinants of fungal-pathogen speciation and host adaptation. Proc Natl Acad Sci U S A. 2014;111(47):16796-801.

22. Larriba E, Jaime MD, Carbonell-Caballero J, Conesa A, Dopazo J, Nislow C, et al. Sequencing and functional analysis of the genome of a nematode egg-parasitic fungus. Pochonia chlamydosporia Fungal Genet Biol. 2014;65:69-80.

23. Bonants PJ, Fitters PF, Thijs H, den Belder E, Waalwijk C, Henfling JW. A basic serine protease from Paecilomyces lilacinus with biological activity against Meloidogyne hapla eggs. Microbiol. 1995;141(Pt 4):775-84.

24. Khan A, Williams K, Molloy MP, Nevalainen H. Purification and characterization of a serine protease and chitinases from Paecilomyces lilacinus and detection of chitinase activity on 2D gels. Protein Expr Purif. 2003;32(2):210-20

25. Maker: http://www.yandell-lab.org/software/maker.html. Accessed 13 Jan 2015

26. Parra G, Bradnam K, Korf I. CEGMA: a pipeline to accurately annotate core genes in eukaryotic genomes. Bioinformatics. 2007;23(9):1061-7.

27. Parra G, Bradnam K, Ning Z, Keane T, Korf I. Assessing the gene space in draft genomes. Nucleic Acids Res. 2009;37(1):289-97.

28. Lowe TM, Eddy SR. tRNAscan-SE: a program for improved detection of transfer RNA genes in genomic sequence. Nucleic Acids Res. 1997;25(5): 955-64.

29. Schattner P, Brooks AN, Lowe TM. The tRNAscan-SE, snoscan and snoGPS web servers for the detection of tRNAs and snoRNAs. Nucleic Acids Res. 2005;1(33(Web Server issue)):W686-9.

30. Mohanta TK, Bae H. The diversity of fungal genome. Biol Proced Online. 2015;17:8.

31. Robbertse B, Yoder RJ, Boyd A, Reeves J, Spatafora JW. Hal: an automated pipeline for phylogenetic analyses of genomic data. PLoS Curr. 2011;3:RRN1213.

32. Sung GH, Hywel-Jones NL, Sung JM, Luangsa-Ard JJ, Shrestha B, Spatafora JW. Phylogenetic classification of Cordyceps and the clavicipitaceous fungi. Stud Mycol. 2007;57:5-59.

33. Rodríguez-Ezpeleta N, Brinkmann $\mathrm{H}$, Roure B, Lartillot N, Lang BF, Philippe $H$. Detecting and overcoming systematic errors in genome-scale phylogenies. Syst Biol. 2007;56(3):389-99.

34. Xiao G, Ying SH, Zheng P, Wang ZL, Zhang S, Xie XQ, et al. Genomic perspectives on the evolution of fungal entomopathogenicity in Beauveria bassiana. Sci Rep. 2012;2:483.

35. PHI-base: The Pathogen - Host Interaction Database: (http://www.phi-base. org/). Accessed 25 Dec 2014.

36. Cuomo CA, Güldener U, Xu JR, Trail F, Turgeon BG, Di Pietro A, et al. The Fusarium graminearum genome reveals a link between localized polymorphism and pathogen specialization. Science. 2007;317((5843):1400-2.

37. Galagan JE, Calvo SE, Borkovich KA, Selker EU, Read ND, Jaffe D, et al. The genome sequence of the filamentous fungus Neurospora crassa. Nature. 2003;422(6934):859-68

38. Sales-Campos H, Tonani L, Cardoso CR, Kress MR. The immune interplay between the host and the pathogen in Aspergillus fumigatus lung infection. Biomed Res Int. 2013;2013:693023.

39. Solomon PS, Tan KC, Sanchez P, Cooper RM, Oliver RP. The disruption of a Galpha subunit sheds new light on the pathogenicity of Stagonospora nodorum on wheat. Mol Plant Microbe Interact. 2004;17(5):456-66.

40. Kulkarni RD, Thon MR, Pan H, Dean RA. Novel G-protein-coupled receptorlike proteins in the plant pathogenic fungus Magnaporthe grisea. Genome Biol. 2005;6(3):R24.

41. Lin $\mathrm{CH}$, Chung KR. Specialized and shared functions of the histidine kinase- and HOG1 MAP kinase-mediated signaling pathways in Alternaria alternata, a filamentous fungal pathogen of citrus. Fungal Genet Biol. 2010; $47: 818-27$.
42. Morschhäuser J. Regulation of multidrug resistance in pathogenic fungi. Fungal Genet Biol. 2010;47(2):94-106.

43. Moktali V, Park J, Fedorova-Abrams ND, Park B, Choi J, Lee YH, et al. Systematic and searchable classification of cytochrome P450 proteins encoded by fungal and oomycete genomes. BMC Genomics. 2012;13:525.

44. Kelkar HS, Skloss TW, Haw JF, Keller NP, Adams TH. Aspergillus nidulans stcL encodes a putative cytochrome P-450 monooxygenase required for bisfuran desaturation during aflatoxin/sterigmatocystin biosynthesis. J Biol Chem. 1997;272(3):1589-94.

45. Barriuso J, Nguyen DT, Li JW, Roberts JN, MacNevin G, Chaytor JL, et al. Double oxidation of the cyclic nonaketide dihydromonacolin $L$ to monacolin J by a single cytochrome P450 monooxygenase. LovA J Am Chem Soc. 2011;133(21):8078-81.

46. Rawlings ND, Morton FR, Kok CY, Kong J, Barrett AJ. MEROPS: the peptidase database. Nucleic Acids Res. 2008;36(Database issue):D320-5.

47. Rawlings ND, Morton FR. The MEROPS batch BLAST: a tool to detect peptidases and their non-peptidase homologues in a genome. Biochimie. 2008;90(2):243-59.

48. Li J, Yu L, Yang J, Dong L, Tian B, Yu Z, et al. New insights into the evolution of subtilisin-like serine protease genes in Pezizomycotina. BMC Evol Biol. 2010;10:68.

49. Larriba E, Martín-Nieto J, Lopez-Llorca LV. Gene cloning, molecular modeling, and phylogenetics of serine protease P32 and serine carboxypeptidase SCP1 from nematophagous fungi Pochonia rubescens and Pochonia chlamydosporia. Can J Microbiol. 2012;58(7):815-27. doi:10. 1139/w2012-054

50. Cazy: Carbohydrate-Active enZYmes Database: (http://www.cazy.org/). Accessed 5 Jan 2015

51. Hmmer: biosequence analysis using profile hidden Markov models: (http:// hmmer.janelia.org/). Accessed 8 Jan 2015.

52. Zhao Z, Liu H, Wang C, Xu JR. Comparative analysis of fungal genomes reveals different plant cell wall degrading capacity in fungi. BMC Genomics. 2013;14:274

53. Mikami Y, Yazawa K, Fukushima K, Arai T, Udagawa S, Samson RA. Paecilotoxin production in clinical or terrestrial isolates of Paecilomyces lilacinus strains. Mycopathologia. 1989;08(3):195-9.

54. SMURF: Secondary Metabolite Unique Regions Finder: (www.jcvi.org/smurf/ index.php). Accessed 5 Jan 2015.

55. AntiSMASH: antibiotics \& Secondary Metabolite Analysis SHell: (http:// antismash.secondarymetabolites.org/). Accessed 11 Jan 2015.

56. Sharma S, Adholeya A. Detoxification and accumulation of chromium from tannery effluent and spent chrome effluent by Paecilomyces lilacinus fungi. Int Biodeterior Biodegradation. 2011;65:309e317.

57. AllpathsLG: High quality genome assembly from low cost data: (http:// www.broadinstitute.org/software/allpaths-lg/blog/). Accessed 13 Jan 2015.

58. SNAP: http://www.broadinstitute.org/mpg/snap/. Accessed 13 Jan 2015.

59. Genemarkhmm Georgia Institute of Technology, Atlanta, Georgia, USA (http://exon.gatech.edu/GeneMark/). Accessed 16 Jan 2015.

60. Augustus: gene prediction (http://augustus.gobics.de/). Accessed 20 Jan 2015.

61. AFA, Smit, R. Hubley P. Green RepeatMasker at http://repeatmasker.org.

62. Jurka J, Kapitonov W, Pavlicek A, Klonowski P, Kohany O, Walichiewicz J. Repbase Update, a database of eukaryotic repetitive elements. Cytogenet Genome Res. 2005;110:462-7.

63. Chikhi R, Medvedev P. Informed and automated k-mer size selection for genome assembly. Bioinformatics. 2014;30(1):31-7.

64. Zhang Z, Carriero N, Zheng D, Karro J, Harrison PM, Gerstein M. PseudoPipe: an automated pseudogene identification pipeline. Bioinformatics. 2006; 22(12):1437-9.

65. BLAST: Basic Local Alignment Search Tool at National Center for Biotechnology Information (NCBI). http://blast.ncbi.nlm.nih.gov/Blast/. Accessed 28 Dec 2015

66. InterProScan: protein sequence analysis \& classification. (http://www.ebi.ac. uk/interpro/). Accessed 24 Jan 2015.

67. Blast2GO. http://www.blast2go.com/b2ghome/. Accessed 29 Jan 2015.

68. SignalP 4.1 Server. http://www.cbs.dtu.dk/services/SignalP. Accessed 2 Feb 2015.

69. GPCRDB: information system for G protein-coupled receptors: (http://www. gpcr.org/7tm/). Accessed 5 Feb 2015.

70. TMHMM Server v. 2.0: Prediction of transmembrane helices in proteins. (http://www.cbs.dtu.dk/services/TMHMM/). Accessed 5 Feb 2015. 
71. Enright AJ, Van Dongen S, Ouzounis CA. An efficient algorithm for largescale detection of protein families. Nucleic Acids Res. 2002;30(7):1575-84

72. Markov Cluster Algorithm (MCL). http://micans.org/mcl/. Accessed 6 Feb 2015.

73. Muscle: Multiple Sequence Alignment. (http://www.ebi.ac.uk/Tools/msa/ muscle/). Accessed 10 Feb 2015.

74. Gblocks: Castresana Lab, Animal Biodiversity and Evolution Program. (http:// molevol.cmima.csic.es/castresana/Gblocks.html/). Accessed 13 Feb 2015.

75. Fasconcat. (http://software.zfmk.de). Accessed 16 Feb 2015.

76. RaxML. (http://sco.h-its.org/exelixis/software.html). Accessed 18 Feb 2015.

Submit your next manuscript to BioMed Central and we will help you at every step:

- We accept pre-submission inquiries

- Our selector tool helps you to find the most relevant journal

- We provide round the clock customer support

- Convenient online submission

- Thorough peer review

- Inclusion in PubMed and all major indexing services

- Maximum visibility for your research 